\title{
A Short Review of Some of the Factors Affecting the Breakdown Strength of Insulating Oil for Power Transformers
}

\author{
Michael G. Danikas \\ Democritus University of Thrace, \\ Dpt Department of Electrical and \\ Computer Engineering, Power \\ Systems Laboratory, Xanthi, Greece \\ mdanikas@ee.duth.gr
}

\author{
Ramanujam Sarathi \\ Indian Institute of Technology \\ Madras, Department of Electrical \\ Engineering, High Voltage \\ Laboratory, Chennai, India \\ rsarathi@iitm.ac.in
}

\author{
Sayidul Morsalin \\ University of New South Wales, \\ School of Electrical Engineering and \\ Telecommunications, High Voltage \\ Laboratory, Sydney, Australia \\ sayidul.morsalin@unsw.edu.au
}

\begin{abstract}
The present short review investigates some of the factors affecting the breakdown strength of transformer oil. Factors such as the conditioning effect, effect of oil flow, influence of impurities, type of applied voltage and electrode configuration and geometry are further discussed. It should be noted that this review does not discuss the effect of factors such as area, volume and capacitance. Velocity, impurities, electrodes' shape and the type of applied voltage are among the factors documented to have a significant effect on breakdown strength.
\end{abstract}

Keywords-transformer oil; breakdown strength; velocity; flow; electrodes; geometry; configuration

\section{INTRODUCTION}

Liquid dielectrics are used as insulating media in high voltage systems as they can easily fill any space and can also be circulated to dissipate heat. Their main disadvantage is that they get contaminated over time/use. Oil, of certain type, is one of the most frequently used liquids for electrical insulation, frequently referred to as transformer oil due to its wide use within transformers. Previous research was concerned with attempts to understand the physics of breakdown in transformer oil $[1,2]$ as well as with efforts to elucidate the various factors affecting its breakdown strength [3]. The breakdown strength of transformer oil in a lab, depends on a variety of factors either due to the test conditions or to the oil itself. Thus, the breakdown strength of the oil is something which cannot be defined simply by its arithmetic value. Test conditions have to be fully and accurately described. Since most practical insulating liquids depart from the ideal liquid state, it is convenient to call "weak links" all the departures from the ideal state. The breakdown mechanism is assumed to be initiated by a 'weak link', i.e. all sorts of irregularities existing either on the electrode surfaces or in the liquid itself. An increase to the number of "weak links' results to an increase of the probability for breakdown at a given stress. An increase in the size of specimen implies therefore a reduction in breakdown strength. This is the so-called size effect [4].

Transformer oil is used as insulating medium for many decades. Early work in the field of breakdown of liquid dielectrics was done many decades ago [5]. A comprehensive review on breakdown of insulating liquids was also published [6]. It is generally agreed that electrical breakdown in liquids is statistical in nature and the breakdown strength depends on additives, pressure, field geometry, size of the whole system, electrode material and finish, impurities, voltage waveform, past history, time and magnitude of pre-stress. It is the purpose of the present short review to give some aspects of the research performed over the past years regarding some of the factors affecting the breakdown strength of transformer oil.

\section{THE CONDITIONING EFFECT}

The conditioning process is the process according to which a change in strength is obtained with successive breakdowns. Quite early a gradual increase in the breakdown strength with increasing number of measurements was observed. A lack of conditioning effect in $n$-hexane was probably due to the fact that particles larger than $10 \mu \mathrm{m}$ were removed by filtration [7]. Other researchers showed that the conditioning process varied for different electrode metals and it also depended on the treatment of the electrode surface. It was pointed out that the conditioning phenomenon was due to the gradual degassing of the electrodes which accompanies the successive discharges [8]. Also, that the conditioning effect was less marked for pure than for impure liquids, agreeing in that with [7]. Other researchers also agreed with [7], attributing the conditioning effect to the removal of moisture and the destruction of fibers by successive breakdowns [9]. Experiments with impulse voltages showed an upward trend, then a plateau value of the conditioned state and finally a downward trend in breakdown strength [10]. Commenting on these findings, some years later it was remarked that the full conditioning effect may be masked by a reduction in strength associated with electrode damage [11]. The conditioning effect is not absent even with the transformer oil circulating between breakdowns. Such experimental data tends to reject theories of fiber or moisture removal [12]. A downward trend of the breakdown strength of an oil sample rested in the middle of a series of conditioning breakdowns was also observed [10], whereas other researchers 
observed an upward trend under similar conditions [13]. The observed downward trend was explained by some researchers, who suggested that the conditioning effect is mainly governed by a dielectric layer on the cathode which plays a part in the breakdown process by acting as a barrier to positive ions which were formed in the gap by electron bombardment of molecules. The positive ions, prevented from reaching the cathode itself, enhance the electric field at the cathode, increasing thus the field emission of electrons [14]. The above process was contested in [11], where it was pointed out that such process can only take place with clean and highly polished electrodes. In [1], another explanation was given for the downward condition, namely that this occurs when a relatively large energy is dissipated at breakdown leading to the melting of a greater quantity of electrode metal and the contamination of the liquid by electrode material and decomposed liquid products, especially if these are not given time to clear from the gap. Such an explanation - regarding the downward trend - is in agreement with the explanation given in $[15,16]$. In accordance with the above is also the model put forward in [17], where the upward or downward trend in conditioning is explained by the creation of an insulating layer on the electrodes (for upward conditioning) and its subsequent destruction (for downward trend) because of chemical transformations.

A slight conditioning effect is also observed with mineral oil using standard Foster test cell electrodes [18]. The authors, however, did not give any particular reason as to why this should be so. Given that their electrodes were polished and clean, one can reasonably assume that possible removal of moisture may be the reason for the slight conditioning effect. Such an interpretation may be supported particularly in view of recent research, which claims that the breakdown strength remains high when moisture saturation is below $20 \%$ but it decreases significantly as relative moisture saturation increases [19]. Conditioning of the electrodes is not observed if the oil is replaced after each breakdown. On the other hand, if the oil is not replaced and the volume is small, previous breakdowns may reduce the breakdown strength because of contamination by products of its decomposition and by the electrode metal [20]. Another factor which has to be taken into account is the settling time between consecutive breakdowns. In this respect, past research as well as more recent work indicate that conditioning is influenced by the settling time, i.e. the longer the time interval between breakdowns, the higher the probability for higher breakdown values [21,22]. This is due to the fact that shorter settling times do not allow either the bubbles from the previous breakdown to dissolve or the decomposition products to settle under the influence of gravity.

\section{THE VELOCITY EFFECT}

Since in contaminated liquids breakdown events are dominated by a variety of phenomena associated with the contamination, it is only logical to suppose that by trying to destroy possible foreign particle bridges between the electrodes, the breakdown strength will increase [1]. If oil is pumped through a gap between the electrodes, or the electrodes are rotated, the breaking of fiber chains, movement of gas accumulations and possibly the disturbance of electrochemical processes at the electrodes, will affect the breakdown strength [1]. Although in [3] no significant change in breakdown strength was observed when circulating oil instead of stationary oil was used, other researchers found that the breakdown strength and the mean gap velocity were related almost linearly for AC testing voltages [11]. However, the latter researcher was cautious as to the effect of oil velocity as there was no information on the variation of oil density, its viscosity and its surface tension over the period of the experiment [11]. Later on, it was noted that the oil flow caused the distributions of breakdown values to be normal instead of extreme, as it was the case with static oil. An enhancement of the breakdown strength was observed when flowing oil was used. It was also noted that normalization takes place gradually with the increase of velocity. It was also remarked that the oil velocity cannot be raised indefinitely because cavitation will eventually cause a reduction of the breakdown strength [23]. The increase of breakdown strength with flowing oil was explained either by the continuous removal of decomposition products from the test gap or by the prevention of particle bridges to be formed in the gap [11]. The flowing oil may also impede the entry of impurities into the test gap [24]. The reported change in breakdown strength with flowing oil is achieved at oil velocities up to $3 \mathrm{~cm} / \mathrm{s}$ [25]. Earlier work indicated that with even lower oil velocities (up to $0.5 \mathrm{~cm} / \mathrm{s}$ ) higher breakdown strength can be achieved [23]. An indefinite increase of breakdown strength is not envisioned since the increase of the oil velocity causes the flow to be turbulent. Turbulent flow will cause the creation of bubbles in the volume of the liquid as a result of the difference in pressure. The approach of [23] and [25] seems to be in agreement with the view that the breakdown strength of the oil increases up to a certain velocity but as soon as this limit is passed, it falls [26]. In fact the limit of $3 \mathrm{~cm} / \mathrm{s}$ discussed in [25] agrees well with the data presented in [26], where a limit of about $5 \mathrm{~cm} / \mathrm{s}$ was observed for AC and DC voltages. The oil flow and its beneficial effects is intimately linked with the presence - and eventual removal - of foreign particles and impurities in the oil. Under both $\mathrm{AC}$ and DC voltages a bridge between the electrodes is highly likely to occur and thus breakdown probability is increased [27]. Talking about foreign particles in oil, it has been indicated that metallic particles tend to lower the breakdown strength more than non-metallic particles $[25,28]$.

\section{THE EFFECT OF IMPURITIES}

Impurities play a critical role in the breakdown of transformer oil $[3,6]$. Impurities can be classified as particles of dust or fibers which are present in the liquid, sometimes escaping the filtration process as being very small, particles produced by previous discharges, such as carbon particles resulting from the decomposition of the liquid or minute metallic particles removed from the electrode surface by high energy sparks, water, additives deliberately introduced into the liquid to change its properties. It has been reported that with voltages applied for a long period, particles or fibers in the bulk of the liquid are attracted to the region of highest stress, forming a bridge and consequently causing a breakdown [9]. Under both AC and DC voltages the addition of even small amounts of water to treated oil causes a significant reduction in the breakdown strength. The reduction is even more 
pronounced when the oil contains already suspended matter [29]. Agreeing with [29], it was indicated that the effect of moisture depends entirely on the nature and the amount of other impurities present in the bulk of the liquid [30]. Moreover, above a certain stress level, $300 \mathrm{kV} / \mathrm{cm}$ according to [31], particle activity may stop because of either an ejection of impurities from the gap or an adhesion to the electrode surfaces. The influence of impurities also depends on the molecular structure of the liquid [32], as foreign particles can be attracted into the gap and form a bridge distorting the electric field between the electrodes [7]. An improvement in breakdown strength can be achieved by increasing the number of filtering cycles of the oil. It has been shown that, by using small size Bruce profile electrodes, the mean breakdown strength can be increased by about $8 \%$ after 5 filtration cycles and by about $10 \%$ after 8 filtration cycles [25]. Such an improvement is probably due to the fact that the distribution of particle sizes moves to smaller sizes as the number of filtration cycles increases. If after a certain number of filtering cycles no change in breakdown strength is observed, this means that extremely small particles pass through the filter in the actual oil volume, limiting thus its breakdown strength.

Metallic particles have a more dramatic effect on breakdown strength than non-metallic particles [25]. The dominant role of impurity particles is due to the possibility of bridge formation and the establishment of convenient nucleation sites for bubble formation [6, 24, 33-36]. Moreover, the propagation rate of pre-breakdown disturbances increases with the contamination of the oil with the consequent effect of the reduction of the formative time lag, which eventually leads to a decrease of the breakdown strength [37]. Carbon particles affect the breakdown strength as was reported in [25], where with small size Bruce profile electrodes and small gaps (1-4 $\mathrm{mm})$ a decrease of breakdown strength was observed up to $12 \%$. More recently, research has shown that the influence of carbon particles on breakdown strength depends also on the type of applied voltage, i.e. with plane-plane electrodes and AC voltages the breakdown strength was higher than with DC voltages. This means that under AC voltages, bridges of particles are more difficult to be formed. On the other hand, a composite $\mathrm{AC} / \mathrm{DC}$ voltage gives breakdown strength results between the $\mathrm{AC}$ voltage and the $\mathrm{DC}$ voltage [38]. It has also been indicated [39] that with DC voltages, point-plane electrodes and pure oil, the breakdown strength was higher with the point electrode negative, whereas with contaminated oil -and especially at higher applied voltages- there was an inversion in experimental results, i.e. the positive point gave the higher breakdown strength. Such an inversion was explained as due to space charges, which may be particularly noticed in contaminated oil [39]. Different kinds of particles (metallic, cellulose and mixed) were investigated with DC voltages and uniform electrode arrangement in [40] and it was observed that mixed (i.e. metallic with cellulose) particles lower indeed the breakdown strength of transformer oil. In fact, mixed particle bridges were the densest between the electrodes. Metallic particles do not form stable bridges since they tend to go to the bottom of the test cell. It is evident that the formation of bridges depends on voltage duration and the type of particles involved. The influence of moisture in combination with foreign particles was noted quite early [29], where it was remarked that the influence of moisture is very harmful when the oil contains particles of various sizes although, as commented in [41], the size of particles plays a more dominant role rather than the high concentration of particles itself. Such observations were confirmed in [42]. Furthermore, in [42] it was pointed out that 5 purification cycles are enough for the oil to be cleaned, something which is in rough agreement with data from [25].

As a general comment, it can be pointed out that foreign particles do indeed lower the breakdown strength of transformer oil, particularly if they are combined with moisture. Inclusions of moisture more than about $10 \mathrm{ppm}$ at normal temperature may be enough to cause a lowering of breakdown strength. On the other hand, high breakdown strength does not necessarily mean that there is an absence of foreign particles. It may well mean that the quantity of foreign particles is not large enough to influence the breakdown strength $[43,44]$.

\section{THE VOLTAGE DURATION EFFECT}

Many phenomena observed in measuring the breakdown strength of insulating liquids are time dependent $[11,23]$. Although the duration of impulse voltages is very short compared to $\mathrm{AC}$ voltages, there were reports confirming that the breakdown strength is independent of the duration of the voltage application for both DC and AC voltages [29]. Breakdown in oil occurs at the tail of the impulse voltage [29]. According to [45], impulse testing furnishes some information about the breakdown mechanism, namely that the breakdown time-lag can be divided into two intervals, the statistical time lag and the formative time lag, pointing out that the latter depends on the nature of the breakdown process and the test conditions. For pure organic liquids the breakdown strength is independent of the pulse duration above a certain time-lag [14, 46]. They explained that by assuming that the formative timelag is associated -at least partly- with the time required for some charge species to cross the gap between the electrodes. In [46], a streamer-like mechanism was suggested, composed by two parts, firstly the time required for a single electron avalanche to produce sufficient field distortion near the anode to favor the growth of the streamer, and secondly the time required for the streamer to cross the gap. It has also been suggested that the breakdown strength is determined by the liquid properties for long pulses and by the emission characteristics of the cathode for short pulses [47]. Agreeing with [46], the authors of [47] suggested that breakdown is initiated by an extra-large localized burst of electrons from the cathode. These bursts are likely to occur at certain active sites where emission momentarily becomes easy, possibly because of irregularities or inclusions or surface deposits.

In analyzing the breakdown process under both constant stress and ramp stress conditions, [48] concluded that statistical procedures allow the formative time lag to be separated from the total breakdown time. Slow ramps cause the smallest breakdown values. Furthermore, [48] indicated that even the slightest alterations in experimental conditions may cause considerable changes in breakdown strength. In yet another publication [49], the same authors showed that for unit step 
waveforms, the mean statistical time lag for breakdown in nhexane is inversely proportional to electrode area for uniform field geometry but independent of the gap spacing. They remarked, agreeing with [47], that there is strong evidence for the location of the initiating event on the electrode surface. Voltage duration affects the particle movement in transformer oil and consequently its breakdown strength as was reported in [50]. DC voltage is more prone to create bridges of iron particles than $\mathrm{AC}$ voltage, the reason being that with $\mathrm{AC}$ voltage iron particles are under the influence of alternative electric forces, which render the lining up more difficult. With pulsating DC voltage the situation is between the two aforementioned cases. What is reported in [50] is in line with the findings of [38]. The results reported in [38] and [50] generally agree with those of [51], where it was shown that with DC or pulses longer than $50 \mathrm{~ms}$ foreign particles can form a bridge that may initiate a breakdown. In [51], it is cautiously suggested that for pulsed conditions at frequencies of up to 100 $\mathrm{Hz}$, one can afford to decrease the insulation thickness by $10 \%$ to $20 \%$ relative to those required at DC voltage. Further evidence in validation of $[38,50,51]$ was offered in [52], where a distinct difference between $\mathrm{DC}$ and $\mathrm{AC}$ voltages regarding the movement of foreign particles in transformer oil was described. According to [51], bridging of foreign particles under DC voltage is encouraged by dielectrophoretic (DEP) forces and as the voltage increases the velocity of particles also increases with the rate of bridge formation intensified. The associated conduction current increases and the bridges become wider but not denser. The effect of impurities with short duration pulses $(\sim 1 \mu \mathrm{s})$, however, is rather small [53].

Generally speaking, it can be said that voltage duration has indeed an effect on the breakdown strength of transformer oil. It is certainly to be noted that fast pulses cause higher breakdown strength than longer pulses or even DC voltages. The effect of voltage duration also depends, on the other hand, also on the quality of the transformer oil tested [1,54]. More contaminated oils are prone to be more sensitive to the type of the applied voltage.

\section{THE EFFECT OF GEOMETRICAL CONFIGURATION OF THE ELECTRODES}

The geometrical configuration affects the degree of the uniformity of the electric field. Electrodes with Bruce (or Rogowski) profiles show approximately uniform field distributions. Uniform field configurations are useful since they give fundamental information on the breakdown parameters. Non-uniform field configurations are also very useful since they supply information about the expected performance of practical devices as was pointed out in [55]. Non-uniform field configuration affects the breakdown mechanism [56], with the authors claiming that -regarding paper/oil insulation- gas evolution is of primary importance with decomposition starting from the decomposition of water in the paper. In [29], the authors using non-uniform field configurations suggested that the initiation of the breakdown can be divided into two parts: one including the formation of gas bubbles in the liquid at the cathode, and the other, being an adequate multiplication by a gaseous ionization in the bulk of the liquid. They also suggested that the formation of bubbles depends on the magnitude of the electric field, on the external pressure and on the amount of gas available at or near the electrodes. Polarity effect is affected by the gas content of the liquid as was pointed out in [57]. Uniform field configurations are used on many occasions in order to see the relation between the applied voltage and the gap spacing. According to [58], the mean breakdown voltage $(\mathrm{V})$ varies linearly with the logarithm of gap spacing (d) following the general formula below $\mathrm{V}=\mathrm{Kd}^{\mathrm{n}}$, where, $\mathrm{n}$ takes values between 0 and 1 . Such a relation was also proposed in [59], where it was noted that the breakdown voltage increase is somehow less proportional to the gap length for uniform field electrodes (plane-plane arrangement). Experimenting with different electrode shapes, some researchers reached the conclusion that foreign particle bridge is thick and strongly bonded for spherical electrodes under DC voltage, whereas a thinner bridge is formed with needle-plane electrodes under DC voltage. This can be explained by considering that the DEP force is very strong in the first case but only strong near the needle electrode in the second case [60]. Such observations are in agreement with those of [52]. Somewhat similar results were obtained in [61], where it was remarked that gas bubbles have a minimum effect with a nonuniform field arrangement because the movement of the bubbles and the corona will prevent the bubbles' discharge path. Discharge characteristics have been investigated in [62] with different electrode arrangements varying from point-plane to semi-homogeneous sphere-plane configuration, where it was reported that they depend on applied voltage, time of application and moisture content. Non-uniform electrodes help in defining the various modes of streamer propagation in oils. Earlier research indicated that the breakdown mechanism is better studied with a point-plane arrangement and it was indicated that there are three modes, with modes 2 and 3 having velocities $3 \mathrm{~km} / \mathrm{s}$ and $10 \mathrm{~km} / \mathrm{s}$ respectively [63, 64]. Such streamer modes, albeit with different velocities, were also recorded in [65], where experiments with plane-plane electrodes were performed with a combination of mineral oil and solid spacers.

It is evident that electrode geometry affects greatly the breakdown process and strength of transformer oil. Things become even more complicated because far too often there are bubbles and minute foreign particles in an oil gap [51, 61]. Especially, non-uniform electrode arrangements may indicate the breakdown streamer mechanism in an oil gap [66, 67].

\section{SOME GENERAL REMARKS}

This short review points out some of the factors affecting the breakdown strength in transformer oil. Certainly, not all of the factors were described and analyzed. A volume effect and/or an area effect may result if weak links are present in the liquid itself and/or on the electrode surfaces [68, 69]. Earlier on, it was shown that with impulse voltages a simple volume effect may exist since the area and gap effects were approximately equal but for $\mathrm{AC}$ voltages the breakdown strength dependence on gap spacing was found to be much greater than on area [3]. Experimental data also confirmed this [25]. Such a stronger dependence on gap length may be traced back for explanation to [70], where it was reported that the charge of a particle in contact with an electrode is proportional 
to the gap voltage and not to the local applied field. No comment was offered in the context of the present review about the capacitance effect, namely the effect according to which by putting a capacitance of known value in parallel to the test cell of the electrodes the conditioning effect may be altered. There is an optimum value of capacitance which, by delivering the right amount of energy into the electrode gap, may improve this process by destroying the most prominent irregularities on the electrode surfaces $[25,71]$. Regarding some future work, it would be of great interest if the role of bubbles and/or foreign particles could be studied in relation to nanofluids since the latter show some promise for replacement of the more traditional liquids $[72,73]$. The so-called velocity effect is also a promising field of work w.r.t. the nanofluids as well as to the proposed ester and vegetable oils $[74,75]$.

\section{CONCLUSIONS}

In this short review, some factors affecting the breakdown strength of transformer oil were referred to. The main factors considered are the oil velocity effect, the presence of impurities, the conditioning and shape of the electrodes and the type of applied voltage.

\section{REFERENCES}

[1] D. F. Binns, "Breakdown in liquids", in: Electrical Insulation, Peter Peregrinus Ltd., London, UK, pp. 15-32, 1983

[2] K. C. Kao, "Theory of high-field electric conduction and breakdown in dielectric liquids", IEEE Transactions on Electrical Insulation, Vol. 11, No. 4, pp. 121-128, 1976

[3] W. R. Bell, "Influence of specimen size on the dielectric strength of transformer oil", IEEE Transactions on Electrical Insulation, Vol. 12, No. 4, pp. 281-292, 1977

[4] B. N. Taylor (ed), Journal of Research of the National Institute of Standards and Technology, Special Issue-Extreme Value Theory, Vol. 99, No. 4, July-August 1994

[5] S. Whitehead, Electrical Discharges in Liquids, Eds. Benn, London, UK, 1928

[6] A. H. Sharbaugh, J. C. Devins, S. J. Rzad, "Progress in the field of electric breakdown in dielectric liquids", IEEE Transactions on Electrical Insulation, Vol. 13, No. 4, pp. 249-276, 1978

[7] B. Salvage, "The dielectric breakdown of some simple organic liquids", Proceedings of the IEE, Vol. 98, Part IV, pp. 15-22, 1951

[8] J. L. Maksiejewski, H. Tropper, "Some factors affecting the measurement of the electric strengrh of organic liquids", Proceedings of the IEE, Vol. 101, Part II, pp. 183-190, 1954

[9] P. K. Watson, J. B. Higham, "Electric breakdown of transformer oil", Proceedings of the IEE, Vol. 100, Part IIA, pp. 168-174, 1953

[10] R. Hancox, H. Tropper, "The breakdown of transformer oil under impulse voltages", Proceedings of the IEE, Vol. 105A, pp. 250-262, 1958

[11] J. K. Nelson, "Statistical effects of environmental factors on liquid breakdown measurements", Proceedings of the IEE, Vol. 118, No. 11, pp. $1675-1678,1971$

[12] A. M. Sletten, T. J. Lewis, "The influence of dissolved gases on the electric strength of n-hexane", British Journal of Applied Physics, Vol. 14, No. 12, pp. 883-888, 1963

[13] A. A. Zaky, M. E. Zein Eldine, R. Hawley, "The electric strength of mineral oil using bare and coated electrodes", British Journal of Applied Physics, Vol. 16, No. 4, pp. 437-440, 1965

[14] K. C. Kao, J. B. Higham, "The effects of hydrostatic pressure, temperature and voltage duration on the electric strengths of hydrocarbon liquids", Journal of Electrochemical Society, Vol. 108, No. 6, pp. 522-528, 1961
[15] W. R. Bell, M. Danikas, "Factors affecting the breakdown strength of transformer oil", Conference Record of 1982 IEEE International Symposium on Electrical Insulation, Philadelphia, PA, USA, pp. 264267, June 7-9, 1982

[16] M. G. Danikas, "Breakdown of transformer oil", IEEE Electrical Insulation Magazine, Vol. 6, No. 5, pp. 27-34, 1990

[17] J. Fuhr, W. F. Schmidt, "Spark breakdown of liquid hydrocarbons - II. temporal development of the electric spark resistance in n-pentane, $n$ hexane, 2,2 - dimethylbutane, and n-decane", Journal of Applied Physics, Vol. 59, No. 11, pp. 3702-3708, 1986

[18] Y. Jing, I. V. Timoshkin, M. P. Wilson, M. J. Given, S. J. MacGregor, T. Wang, "Dielectric properties of natural ester, synthetic ester Midel 7131 and mineral oil Diala D", IEEE Transactions on Dielectrics and Electrical Insulation, Vol. 21, No. 2, pp. 644-652, 2014

[19] Vaisala, "The Effect of Moisture on the Breakdown Voltage of Transformer Oil" available at: https://www.vaisala.com/sites/default/ files/documents/CEN-TIA-power-whitepaper-Moisture-and-BreakdownVoltage-B211282EN-A-LOW.pdf

[20] V. Y. Ushakov, Insulation of High-Voltage Equipment, Eds. SpringerVerlag, Heidelberg, Germany, 2004

[21] M. G. Danikas, "Particles in transformer oil", IEEE Electrical Insulation Magazine, Vol. 7, No. 2, pp. 39-40, 1991

[22] S. A. Ward, "Performance of particles and water contamination on the dielectric strength of transformer oils", $1^{\text {st }}$ Baha Technical Meeting (BTM 2004), Al Baha, Saudi Arabia, pp. 421-433, 2004

[23] J. K. Nelson, B. Salvage, W. A. Sharpley, "Electric strength of transformer oil for large electrode areas", Proceedings of the IEE, Vol. 118, No. 2, pp. 388-393, 1971

[24] N. D. Clothier, "Solid particle impurities in power transformers", The Electrical Review, Vol. 186, pp. 648-650, 1970

[25] M. G. Danikas, Factors affecting the breakdown strength of transformer oil, M. Sc. Thesis, University of Newcastle-upon-Tyne, Department of Electrical and Electronic Engineering, UK, 1982

[26] M. Ikeda, T. Teranishi, M. Honda, T. Yanari, "Breakdown characteristics of moving transformer oil", IEEE Transactions on Power, Apparatus and Systems, Vol. 100, pp. 921-928, 1981

[27] M. M. Alharthi, S. S. M. Ghoneim, I. B. M. Taha, "Breakdown voltage of the Transformer oils under certain conditions", International Journal of Applied Engineering Research, Vol. 13, No. 6, pp. 3810-3815, 2018

[28] S. S. Sinan, J. Jasni, N. Azis, M. Z. A Ab Kadir, M. N. Mohtar, "Effect of particles on the AC breakdown voltage of palm oil and coconut oil", Applied Mechanics and Materials, Vol. 793, pp. 70-74, 2015

[29] M. E. Zein Eldine, H. Tropper, "The electric strength of transformer oil", Proceedings of the IEE, Vol. 103, pp. 35-45, 1956

[30] E. T. Norris, "High-voltage power-transformer insulation", Proceedings of the IEE, Vol. 110, No. 2, pp. 428-440, 1963

[31] A. M. Z. Huq, H. Tropper, "Conduction current pulses in organic insulating liquids under electrical stress", British Journal of Applied Physics, Vol. 15, No. 5, pp. 481-490, 1964

[32] K. Yoshino, "Dependence of dielectric breakdown of liquids on molecular structure", IEEE Transactions on Electrical Insulation, Vol. 15, No. 3, pp. 186-200, 1980

[33] J. Skowronski, B. Lutynski, "New system of spark-gap electrodes for measurement of dielectric strength of insulating liquids", Proceedings of the IEE, Vol. 113, No. 6, pp. 1106-1108, 1966

[34] J. Crawley, L. Angerer, "Investigation of the electrical breakdown of transformer oil in Skowronski's cup-sphere spark gap", Proceedings of the IEE, Vol. 113, No. 6, pp. 1103-1105, 1966

[35] A.-R. Nosseir, "Effect of dissolved gases, stress and gap spacing on high-field conductivity in liquid insulants", IEEE Transactions on Electrical Insulation, Vol. 10, No. 2, pp. 58-62, 1975

[36] A.-R. Nosseir, S. El-Debeiky, I. F. Hashad, H. A-Bary, "Effect of temperature on the breakdown probability of liquid dielectrics", IEEE Transactions on Electrical Insulation, Vol. 15, No. 6, pp. 502-505, 1980

[37] R. N. Allan, E. M. Hizal, "Pre-breakdown phenomena in transformer oil subjected to non-uniform fields", Proceedings of the IEE, Vol. 121, No. 3, pp. 227-231, 1974 
[38] C. Liang, H. Wu, Y. Li, L. Huang, "Influence of impurity concentration on insulation strength of insulating oil under different voltage types", 2nd International Conference on Artificial Intelligence and Engineering Applications (AIEA 2017), pp. 457-464, 2017

[39] J. Fleszynski, B. Lutynski, J. I. Sjkowronski, "Effect of impurities on the breakdown of insulating oil in long gaps", Journal of Electrostatics, Vol. 7, pp. 47-55, 1979

[40] M. Dan, J. Hao, R. Liao, L. Cheng, J. Zhang, F. Li, “Accumulation behaviors of different particles and effects on the breakdown properties of mineral oil under DC voltage", Energies, Vol. 12, Art. No. 2301, 2019

[41] K. N. Mathes, J. M. Atkins, "Influence of particles on partial discharges and breakdown in oil", Conference Record of 1978 IEEE International Symposium on Electrical Insulation, Conf. Record 78 CH 1287-2-EI, Philadelphia, pp. 1-6, 1978

[42] S. Salvi, A. P. Paranjape, "Study of transformer oil purification", SSRG International Journal of Electrical and Electronic Engineering, Vol. 4, No. 3, pp. 16-19, 2017

[43] B. Pahlavanpour, M. Eklund, K. Sundkvist, "Revised IEC standard for maintenance of in-service insulating oil", http://weidmannsolutions.cn/huiyi/Seminar\%202004\%20Sacramento/PahlavanpourPaper 2004.pdf

[44] M. G. Danikas, S. Georghiou, I. Liapis, A. B. B. Abd. Ghani, "Diagnostic techniques in transformer oils: Factors affecting the lifetime of transformer oil in transformers of $150 / 20 \mathrm{kV}$ and the problem of relating diagnostics data with their pre-history", Funktechnikplus\#Journal, Vol. 1, No. 6, pp. 27-40, 2015

[45] T. J. Lewis, "The electric strength of silicone liquids", Proceedings of the IEE, Vol. 104, No. 17, pp. 493-497, 1957

[46] R. W. Crowe, "Formative time lags in the electric breakdown of liquid hydrocarbons", Journal of Applied Physics, Vol. 27, pp. 156-160, 1956

[47] T. J. Gallagher, T. J. Lewis, "Statistical effects in the breakdown of liquid argon under pulse voltages", British Journal of Applied Physics, Vol. 15, No. 8, pp. 929-934, 1964

[48] J. E. Brignell, "Statistical nature of breakdown sequences in a liquid insulant", Proceedings of the IEE, Vol. 113, No. 10, pp. 1683-1691, 1966

[49] J. E. Brignell, K. D. Metzmacher, "Dependence of the statistical breakdown time-lag of a liquid dielectric on electrode geometry", Journal of Physics D: Applied Physics, Vol. 4, No. 2, pp. 253-258, 1971

[50] J. Zhang, J. Li, Y. Wang, Y. Li, X. Li, "Breakdown voltage of transformer oil contaminated with iron particles at pulsating DC voltage", Electric Power Components and Systems, Vol. 46, No. 11-12, pp. 1321-1329, 2018

[51] A. Pokryvailo, C. Carp, "Comparison of the dielectric strength of transformer oil under DC and repetitive multimillisecond pulses", IEEE Electrical Insulation Magazine, Vol. 28, No. 3, pp. 40-49, 2012

[52] S. Mahmud, Influence of contamination on the electrical performance of power transformer oil, Ph. D. Thesis, University of Southampton, Faculty of Physical Sciences and Engineering, School of Electronics and Computer Science, 2015

[53] D. M. Srinivasa, B. Chandrakanth, F. Valentina, B. Pooja, S. Suneel, "Comparative study of breakdown phenomena and viscosity in liquid dielectrics", International Journal of Innovative Research in Electrical, Electronics, Instrumentation and Control Engineering, Vol. 5, No. 6, pp. 47-52, 2017

[54] T. J. Gallagher, Simple Dielectric Liquids: Mobility, Conduction, and Breakdown, Clarendon Press, Oxford, UK, 1975

[55] W. R. Wilson, A. L. Streater, E. J. Tuohy, "Application of volume theory of Dielectric strength to oil circuit breakers", Transactions of the AIEE, Part III: Power Apparatus and Systems, Vol. 74, No. 3, pp. 677 688,1955

[56] Z. Krasucki, H. F. Church, C. G. Garton, "A new explanation of gas evolution in electrically stressed oil-impregnated paper insulation", Journal of Electrochemical Society, Vol. 107, No. 7, pp. 598-601, 1960
[57] H. Tropper, "The effect of dissolved gases on the electrical conduction and breakdown of insulating oil", Journal of Electrochemical Society, Vol. 108, No. 2, pp. 144-149, 1961

[58] Y. Kawaguchi, H. Murata, M. Ikeda, "Breakdown of transformer oil", Power, Apparatus and Systems, Vol. 91, No. 1, pp. 9-23, 1972

[59] B. E. Gaenger, "The breakdown voltage of oil gaps with high DC voltage", IEEE Transactions on Power, Apparatus and Systems, Vol. 87, No. 10 , pp. $1840-1843,1968$

[60] S. Mahmud, I. O. Golosnoy, G. Chen, G. Wilson, P. Jarman, "Effect of different shapes of electrodes on bridging in contaminated transformer oil”, 2014 Annual Report of Conference on Electrical Insulation and Dielectric Phenomena, Iowa, USA, pp. 114-117, October 19-22, 2014

[61] C. Qin, Y. He, B. Shi, T. Zhao, F. Lv, X. Cheng, "Experimental study on breakdown characteristics of transformer oil influenced by Bubbles", Energies, Vol. 11, No. 3, Art. No. 634, 2018

[62] G. Berg, L. E. Lundgaard, "Discharges in combined transformer oil/paper insulation", Proceedings of 1999 IEEE $13^{\text {th }}$ International Conference on Dielectric Liquids (ICDL '99), Nara, Japan, July 25-28, 1999

[63] G. Massala, O. Lesaint, "A comparison of negative and positive streamers in mineral oil at large gaps, Journal of Physics D: Applied Physics, Vol. 34, pp. 1525-1532, 2001

[64] D. Vukovic, S. Tenbohlen, "Comparative evaluation of breakdown strength of natural esters and mineral oil", Proceedings of $16^{\text {th }}$ International Symposium on High Voltage Engineering, Cape Town, South Africa, August 24-28, 2009

[65] M. P. Wilson, I. V. Timoshkin, M. J. Given, S. J. MacGregor, T. Wang, M. A. Sinclair, K. J. Thomas, J. M. Lehr, "Breakdown in mineral oil: Effect of electrode geometry and rate of voltage rise", IEEE Transactions on Dieletrics and Electrical Insulation, Vol. 19, No. 5, pp. 1657-1664, 2012

[66] A. A. Zaky, A. Nosseir, I. Y. Megahed, C. Evangelou, "Effect of polarity and gap length on the breakdown characteristics of mineral oil for non-uniform fields", Journal pf Physics D: Applied Physics, Vol. 9, pp. L77-L79, 1976

[67] A. Beroual, M. Zahn, R. Badent, K. Kist, A. J. Schwab, H. Yamashita K. Yamazawa, M. Danikas, W. G. Chadband, Y. Torshin, "Propagation and structure of streamers in liquiddielectrics", IEEE Electrical Insulation Magazine, Vol. 14, No. 2, pp. 6-17, 1998

[68] N. Parkman, "Breakdown in composites", in: Electrical Insulation, Peter Peregrinus Ltd., London, UK, 1983

[69] J. K. Nelson, "An assessment of the physical basis for the application of design criteria to dielectric structures", IEEE Transactions on Electrical Insulation, Vol. 24, pp. 835-847, 1989

[70] P. Felsenthal, B. Vonnegut, "Enhanced charge transfer in dielectric fluids containing conducting particles", British Journal of Applied Physics, Vol. 18, pp. 1801-1806, 1967

[71] B. Mazurek, J. D. Cross, “An energy explanation of the area effect in electrical breakdown in vacuum", IEEE Transactions on Electrical Insulation, Vol. 22, No. 3, pp. 341-346, 1987

[72] M. G. Danikas, "Bubbles in insulating liquids: A short review", Engineering, Technology \& Applied Science Research, Vol. 9, No. 6, pp. 4870-4875, 2019

[73] M. G. Danikas, "Breakdown in nanofluids: A short review on experimental results and related mechanisms", Engineering, Technology \& Applied Science Research, Vol. 8, No. 5, pp. 3300-3309, 2018

[74] C. Perrier, M. Ryadi, Y. Bertrand, C. Tran Duy, "Comparison between mineral and ester oils", CIGRE Sessions, Paris, August 22-27, 2010

[75] J. Rouabeh, L. M'barki, A. Hammami, I. Jallouli, A. Driss, "Studies of different Types of insulating oils and their mixtures as an alternative to mineral oil for cooling power transformers", Heliyon, Vol. 5, No. 3, Art. No. e01159, 2019 